\title{
Acculturative Family Distancing (AFD) and Depression in Chinese American Families
}

\author{
Wei-Chin Hwang \\ Claremont McKenna College
}

\author{
Jeffrey J. Wood \\ University of California, Los Angeles
}

\author{
Ken Fujimoto \\ University of Illinois at Chicago
}

\begin{abstract}
Objective: Knowledge of acculturative processes and their impact on immigrant families remains quite limited. Acculturative family distancing (AFD) is the distancing that occurs between immigrant parents and their children and is caused by breakdowns in communication and cultural value differences. It is a more proximal and problem-focused formulation of the acculturation gap and is hypothesized to increase depression via family conflict. Method: Data were collected from 105 Chinese American high school students and their mothers. Rasch modeling was used to refine the AFD measure, and structural equation modeling was used to determine the effects of AFD on youth and maternal depression. Results: Findings indicate that greater AFD was associated with higher depressive symptoms and risk for clinical depression. Family conflict partially mediated this relation for youths, whereas for mothers, AFD directly increased risk for depression. Greater mother-child heritage enculturation discrepancies were associated with greater mother and child AFD. Mainstream acculturation discrepancies and language gaps between mothers and youths were not significantly associated with any of the primary outcome variables. Conclusions: Results highlight the need for better understanding of how AFD and other acculturationgap phenomena affect immigrant mental health. They also underscore the need for prevention and intervention programs that target communication difficulties and intergenerational cultural value differences.
\end{abstract}

Keywords: acculturation gap, depression, family, Chinese American, acculturative family distancing

According to census reports, foreign-born immigrants currently make up $11.1 \%$ (31 million) of the U.S. population and have grown rapidly over the years (57\% between 1990 and 2000; Larsen, 2004). Despite our quickly diversifying population, people's understanding of how acculturative processes impact ethnic minority families and our ability to develop effective therapeutic interventions remains limited. This is especially problematic given that ethnic minorities may be at greater risk for developing mental and physical illnesses as they acculturate and across multiple generations (Escobar \& Vega, 2000; Kessler et al., 1994). Given that Asian Americans (with Chinese Americans being the largest subgroup, comprising over 2.7 million) are proportionately the fastest growing minority group in the United States (U.S. Census Bureau, 2002), understanding the factors that affect their mental

Wei-Chin Hwang, Department of Psychology, Claremont McKenna College; Jeffrey J. Wood, Department of Education, University of California, Los Angeles; Ken Fujimoto, Department of Education, University of Illinois at Chicago.

This work was supported in part by the National Institute of Mental Health (NIMH) Grant 1R34MH73545-01A2 and in part by the Asian American Center on Disparities Research through NIMH Grant 1P50MH073511-01A2.

Correspondence concerning this article should be addressed to WeiChin Hwang, Department of Psychology, Claremont McKenna College, 850 Columbia Ave., Claremont, CA 91711. E-mail: whwang@cmc.edu health status is of utmost importance. Recent studies have indicated that major depression is a significant problem for the Chinese American adults and youths (Hwang, Chun, Takeuchi, Myers, \& Prabha, 2005; Hwang, Myers, \& Takeuchi, 2000).

In this study, we examined how acculturation-related processes affect family relations and depression among Chinese Americans. Acculturation is generally known as the process whereby immigrants acquire the behavior and attitudes of the host culture (Rogler, Cortes, \& Malgady, 1991). Acculturation research suffers from a number of methodological and measurement limitations (Escobar \& Vega, 2000). For example, there is no uniform conceptualization, operationalization, or method of measuring acculturation. Moreover, acculturation has been assessed in a multitude of ways, including linguistically, demographically (e.g., country of origin, place of birth, and years in the United States), socioculturally (e.g., values, attitudes, beliefs, behaviors, social relations, and individualistic and collectivistic orientation), and psychologically (e.g., personality, identity, and ethnic identity) (Berry, 2003; Bornstein \& Cote, 2006).

Conceptually, acculturation involves the acquisition of the dominant group's cultural beliefs (mainstream acculturation), behaviors, and values and the relinquishment or retention of one's culture of origin. This broader process has traditionally been termed acculturation but actually involves two processes, mainstream acculturation and heritage enculturation, the latter of which is less frequently differentiated or studied. Rather than relinquish 
their culture, many immigrants choose to retain their heritage culture and language (enculturation) and hope to pass their heritage on to their children (Kim \& Omizo, 2006). Because immigrant parents and children grow up in different cultural environments, an acculturation gap is likely to occur where parents and children possess different cultural values and may also be differentially fluent in mainstream and heritage languages. Specifically, parents and children may give up their heritage culture at different speeds and acquire the characteristics of the host culture at different rates.

Greater acculturation gaps (e.g., marked by a significant discrepancy between parents and children in adoption of the cultural practices of the dominant culture) have been associated with increased intergenerational family conflict and decreased family cohesion and satisfaction in Asian Americans (Farver, Narang, \& Bhadha, 2002; Gil \& Vega, 1996; Kwak, 2003; Rosenthal, Ranieri, \& Klimidis, 1996; Sluzki, 1979; Ying, 1999). Unfortunately, there has been little research on how the heritage enculturation gap affects immigrant families. Nevertheless, there is a welldocumented relationship between high levels of intergenerational family dysfunction and poor mental health among immigrant groups (Dinh \& Nguyen, 2006; Greenberger \& Chen, 1996; Lee \& Liu, 2001; Lee, Su, \& Yoshida, 2005; Su, Lee, \& Vang, 2005; Szapocznik \& Kurtines, 1993; Vega, Khoury, Zimmerman, \& Warheit, 1995). Few studies, however, have integrated these two bodies of research in examinations of how acculturation gaps impact family functioning and resultant psychological dysfunction.

In some recent studies, the relationship among the acculturation gap, family conflict, and youth depression and other mental health outcomes has been examined. However, in these studies, acculturation and enculturation gaps were not clearly distinguished. In a small sample split across the United States and Canada, Crane, Ngai, Larson, and Hafen (2005) found that both the parent-child acculturation gap (obtained by subtracting averaged parental acculturation scores from adolescent acculturation scores) and poor family functioning were independently associated with depressive symptoms among North American Chinese adolescents. Costigan and Dokis (2006) recently found that when Chinese Canadian parents were more strongly oriented toward Chinese culture and preferred to speak Chinese, lower levels of Chinese cultural and linguistic involvement by the children was associated with maladjustment. Child maladjustment was not associated with children's acculturation when parents did not adhere strongly to Chinese culture. When examining the relationship between intergenerational conflict and parenting style, youth distress, and acculturation gaps (as measured by a difference score between youths' and mothers' scores), Lim, Yeh, Liang, Lau, and McCabe (2009) found that acculturation gaps were not associated with psychological distress. When they recoded the difference scores into categorical mismatch scores (i.e., creating cutoff scores for acculturative mismatches), problematic acculturative mismatches (i.e., with youths more acculturated than their parents) continued to show a nonsignificant relationship, but benign mismatches (when parents were more acculturated than the youth — a seemingly atypical combination) were found to be significantly associated with distress. Although intergenerational family conflict and parenting styles evidenced a direct association with outcomes, no mediating relationship was found. Kim, Chen, Li, Huang, and Moon (2009) found that Chinese American adolescent perceptions of paternal parenting mediated the relationship between father-adolescent acculturation gap and adolescent depressive symptoms. However, the mediating relationship among mother-adolescent acculturation gap, maternal parenting, and adolescent outcomes was not found to be significant. In this case, Kim et al. measured the acculturation gap by categorizing standardized acculturation scores into low, middle, and high tertiles and then cross-classifying parent and child difference scores into low, medium, and high acculturative discrepancies. Unfortunately, no studies have been conducted on how acculturation gap issues affect parental outcomes.

Because different methodologies and measurements of the acculturation gap were used in the previously described studies, findings were inconsistent and have precluded our drawing strong conclusions from this body of research. Refining different measurement approaches for assessment of acculturation gap phenomena (Birman, 2006) and evaluating their relationship with clinical outcomes such as depression are sorely needed. In addition, more work needs to be done to determine which acculturation gap phenomena are responsible for mental health difficulties so that more clearly specified targets for intervention and prevention can be developed. Although an acculturation gap may set the stage for problem development in the family, a person's level of acculturation and family acculturation gaps may not directly increase or decrease risk for mental health problems. The definition and measurement of acculturation may be too broad (e.g., languages, values, behaviors, identity, beliefs, ethnicity of social networks, and preferences for foods and music) and refined models that identify more proximal risk factors are needed (Hwang, 2006a).

For example, identifiable culture-related mechanisms such as increased acculturative stress, loss of culturally protective factors over time, difficulties in communication due to cultural differences, and conflicted beliefs and value systems might be more directly related to mental health outcomes than more distal and potentially nonproblematic dimensions that are commonly assessed in acculturation measures (e.g., participating in religious holidays, eating ethnic foods, listening to ethnic music, or associating with ethnic peer groups). Another limitation is that researchers commonly create acculturation gap scores by subtracting parent and child scores on such distal (non-problem-related) acculturation assessments. Communication difficulties that may be influenced by differential language fluency, a major problem among family members who grow up in different cultural environments, are also not typically calculated into such acculturation gap scores (Hwang, 2006a). A more proximal approach may be to directly assess parent-child disagreements in beliefs and values, as well as perceived difficulties in communication with the other party. Because all immigrant families are likely to evidence some form of acculturation gap and not all immigrant families develop problems, direct assessment of more proximal mechanisms of risk may help identify targets for clinical intervention.

\section{Acculturative Family Distancing}

Hwang (2006a) recently proposed an integrated theory of acculturative family distancing (AFD). AFD is a more proximal conceptualization of parent-child acculturation-related challenges than general measures of the acculturation gap. AFD is defined as the distancing that occurs between parents and youths as a result of 
communication difficulties and cultural value incongruence. AFD is hypothesized to be exacerbated by parent-child differences in acculturation and enculturation (i.e., the acquisition of mainstream American cultural values and the retention of heritage cultural values). AFD is also affected by parent-child differences in linguistic fluency (i.e., heritage and mainstream language gaps) (Hwang, 2006a; Hwang \& Wood, 2009). AFD and its core domains (communication difficulties and cultural value incongruence) are hypothesized to increase over time and lead to distancing between parents and youths, thereby increasing risk for family conflict. Family conflict, in turn, increases risk for depression and other psychological problems. The degree of struggle is expected to vary depending on the characteristics and circumstances of the family (e.g., age and generational status). For example, parents and youths who were both born outside of the United States might evidence fewer cultural differences than parents who were born outside the United States and youths who were born in the United States. Family conflict is postulated to increase risk for psychological difficulties such as depression for both youths and their parents (Chung, 2001; Lee, Choe, Kim, \& Ngo, 2000; McGoldrick, Giordano, \& Garcia-Preto, 2005). Unfortunately, there continues to be little research on how these variables affect parental mental health outcomes such as depression.

AFD is different from the acculturation-enculturation and linguistic gaps because the latter tend to be general non-problemrelated indices of acculturation gap phenomena, whereas, AFD is a specific and problem-focused construct. Specifically, acculturation gap scores (there continues to be little research on enculturation gaps) have traditionally been measured with individual scores on categories such as participation in cultural activities and holidays, eating the foods and listening to the music of one culture or the other, and the ethnicity of one's social network (Berry, 2003; Bornstein \& Cote, 2006; Suinn, Rickard-Figueroa, Lew, \& Vigil, 1987). The scores of parents and children are then subtracted from each other to create an acculturation or enculturation gap difference score. The AFD cultural value incongruence domain assesses parent- and child-perceived agreement and disagreement on values and beliefs along areas that can lead to conflict (e.g., traditional gender roles, importance of academic success vs. social life, weighting of individual vs. family needs). The distinction between the acculturation-enculturation gap and AFD is important because the food that one eats or the music that one listens to, per se, is not likely to cause family conflict.

No established measures have been created to assess the language gap, and little research has been conducted on how language gaps affect family conflict and mental health. This is important, since nearly all immigrant families evidence some type of language gap (i.e., children who grow up in the United States are often more fluent in English than their parents, who are more fluent in their heritage language), but not all families develop problems (Hwang, 2006a). Language gaps are hypothesized to increase risk for communication difficulties, a core domain of AFD. There is some evidence to suggest that language gaps harm family communication (Liu, Benner, Lau, \& Kim, 2009; Tseng \& Fuligni, 2000; Weaver \& Kim, 2008). Acculturation-enculturation gaps are a reflection of cultural affiliation and can also increase risk for communication difficulties because of cultural difference in expression and communication styles (e.g., direct vs. indirect, verbal vs. nonverbal; Sue, 1990). This complex interrelationship is one of the reasons that cultural value incongruence and communication difficulties are important parts of the AFD construct.

The theory of AFD also postulates that parent-child distancing becomes particularly salient as children transition from adolescence into early adulthood (Hwang, 2006a). Clinical illustrations demonstrating how both dimensions of AFD affect immigrant families have been documented (Hwang, 2006a), and a 46-item measure of AFD has been developed (Hwang \& Wood, 2009). In a previous study of the effects of AFD processes on Asian American and Latino college students, results indicated that higher levels of AFD were associated with higher psychological distress and greater risk for clinical depression. Rates of clinical depression for Asian American and Latino students were $12.7 \%$ and $14.0 \%$, respectively. Family conflict mediated this relation and supported the hypothesized linkages (Hwang \& Wood, 2009). Unfortunately, parental outcomes were not assessed in this study, and the influence of acculturation-enculturation and linguistic gaps was not controlled.

Our aims in the present study were to (a) refine the AFD measure using item response analyses, (b) assess the construct validity of the refined AFD measure in a high school sample, (c) test the theory-based conceptual model of AFD's influence on family conflict and depression, and (d) assess the association of other relevant variables with AFD (i.e., the acculturationenculturation gap, mainstream and heritage language gap, age, and place of birth). We utilized two informants of the target variables, mother and youth reports. We compared a set of models using structural equation modeling to assess construct validity, the relative fit of differing directions of influence among the constructs, and mediational effects. On the basis of previous psychometric research with the AFD instrument, we predicted that the AFD measure used in this study would exhibit adequate construct validity (Hwang \& Wood, 2009). We also hypothesized that, consistent with AFD theory and previous research, AFD would be found to serve as a predictor of youth and maternal depression via family conflict, even after controlling for more distal cultural influences that had not been previously tested (e.g., mainstream acculturation-heritage enculturation and linguistic gaps and generational status). In previous acculturation gap research, mainstream acculturation has not been separated out from heritage enculturation, nor have mainstream and heritage linguistic gaps been differentiated from each other; thus, no a priori hypotheses on how these variables would differentially affect outcomes of interest were proposed. However, on the basis of AFD theory, we hypothesized that larger gaps would increase risk for AFD and that U.S.-born adolescents would evidence higher risk for AFD than foreign-born adolescents, given the potential for larger cultural differences between parents and youth.

\section{Method}

\section{Participants and Procedures}

Two hundred and forty-one Chinese American families responded to fliers distributed at a high school inviting families to participate in a study regarding Chinese American family health. The school is located in the western United States and is predominantly Chinese American. Chinese Americans compose approximately half of the student body, with the next three largest groups 
being non-Hispanic White (21\%), Hispanic/Latino (10\%), and Korean American (5\%). Students were offered two movie tickets for their participation, and parents received $\$ 10$ each. Families that requested additional information were sent consent forms and a survey packet in their language of preference (i.e., English, traditional Chinese, or simplified Chinese). To ensure confidentiality, we provided each family member with his or her own stamped envelope to return the survey. Families were told that in order for families to participate, their child and at least one parent had to fill out the questionnaires. One hundred and twenty-one (50\%) Chinese American families completed the survey and returned it in the mail. Only $70 \%$ of fathers completed the surveys, whereas $93 \%$ of mothers and $100 \%$ of youths completed the surveys. Mother and youth data were used in the analyses to prevent a substantial loss in sample size. Within this subsample, $94 \%$ of the mothers were foreign born. In order to limit our findings to immigrant families, we excluded U.S.-born mothers from the analyses, resulting in a final sample size of 105 .

The youth sample consisted of $53 \%$ females and $47 \%$ males spread across different grade levels (freshmen $=29 \%$, sophomores $=18 \%$, juniors $=28 \%$, seniors $=26 \%$ ) and ages (age 14 years $=27 \%, 15=16 \%, 16=30 \%, 17=25 \%, 18=3 \%)$. Additionally, $91 \%$ of the students completed the surveys in English, and $49 \%$ of the mothers completed the surveys in Chinese. Fifty-one percent of the students were U.S. born, and the mean length of time in the United States for foreign-born students was 7.04 years $(S D=3.93$, range $=0-16$ years $)$. Mothers were born in a variety of countries, including Taiwan (38\%), mainland China (32\%), Hong Kong (12\%), Vietnam (5\%), Burma (4\%), Thailand $(2 \%)$, and the remainder from other places.

\section{Measures}

Questionnaires were translated and back-translated by several bilingual Chinese-English speakers from various Chinesespeaking areas (e.g., Hong Kong, mainland China, and Taiwan) and were then reviewed by community members and the principal investigator for linguistic comprehensibility. Chinese languages (e.g., Cantonese, Mandarin) are written in the same characterbased writing system. However, different regions (e.g., Hong Kong, mainland China, and Taiwan) may use either traditional or simplified versions of written Chinese (Chinese characters have been simplified over time, and some regions retain use of more traditional writing). Mothers and youths completed all of the measures in either Chinese or English.

Acculturative Family Distancing (AFD) scale. The original AFD scale was a 46-item self-report measure of the two dimensions of AFD, communication difficulties and incongruent cultural values (Hwang, 2006b; Hwang \& Wood, 2009). The two primary dimensions were theoretically derived by the first author on the basis of his clinical experiences with immigrant families and a review of the acculturation literature. AFD items were developed by the first author and later refined through focus groups conducted with a multicultural team of 10 undergraduate and graduate students. Changes were made to the wording of questions to facilitate clarity, and several items were dropped or added to the scale to improve face validity of the construct. A previous study on a preliminary version of the AFD measure indicated that both dimensions evidenced good internal consistency $(\alpha s=.90$ and
.94) and concurrent validity, as evidenced by moderate to large linkages with family conflict and subjective distress in a sample of 186 Asian American and Latino college students (see Hwang \& Wood, 2009).

In this study, the psychometric properties of the AFD rating instrument were examined and the measure was refined with the aid of Rasch modeling. Analyses were conducted to define the ideal dimensionality, identify the ideal rating response structure, and reduce items to only those that uniquely and best estimated a person's AFD level. A Rasch model is a model-based measurement system in which latent trait levels are estimated from the responses a person provides to a set of items and the item difficulty levels of those items (Embretson \& Reise, 2000). Rasch modeling was chosen over other item response models because the item difficulty levels estimated with these models are more meaningful (Wilson, 2005), and they permit examination of the rating scale structure (Andrich, 1996). The Rasch rating scale model (Andrich, 1978) within the framework of the multidimensional random coefficient multinomial logit model (Adams, Wilson, \& Wang, 1997) was employed. ConQuest 2.0 software (Wu, Adams, Wilson, \& Haldane, 2007) was used for all Rasch analyses Adjustments were first made to the dimensional structure of the AFD instrument More detailed information of the analyses can be found in Fujimoto, Hwang, and Wood (2010).

Review of the AFD items indicated that there was the potential for a four-dimensional structure (two Communication subscales and two Values subscales), and Rasch analysis confirmed that four dimensions fit the data better than two dimensions, $\chi_{\text {diff }}^{2}(17)=$ $1688.47, p<.001$. Under the new structure, the communication dimension was refined into two subscales, Effective Communication (EC; Rasch reliability $R=.88$ ), which consisted of seven items (e.g., "I can communicate effectively with my parents"; "I talk with my parents a lot"), and Communication Barriers (CB; $R=.85$ ), which consisted of five items (e.g., "I feel like there is a communication barrier between me and my parents"; "Although I can get my basic points across, it is hard for me to talk about things in greater depth with my parents"). The values dimension was refined into two subscales, Values Agreement (VA; $R=.86$ ), which consisted of 10 items (e.g., "My parents and I share the same values"; "My parents and I agree on the relative importance between academic vs. social life"), and Values Disagreement (VD; $R=.75$ ), which consisted of seven items (e.g., "My parents and I disagree on the importance of having a social life"; "My parents and I disagree on the roles that men and women should have"). Misfitted items (i.e., those not homologous with the other items in the dimension) or overfitted items (i.e., those redundant with other items in a dimension) were removed. The reduction of items proceeded in an iterative fashion; one item was removed at a time (items with zinfit values greater than 2.0 were considered to be misfitted and items with values less than -2.0 were considered overfitted; Wolfe \& Smith, 2007). Then we refitted the Rasch model. Tests of convergent and discriminant validity are presented in the Results section. The parent version was modified such that the word "parents" was replaced with "child," with concordant changes in verb conjugation.

Next, the rating category structure was modified. Items were originally rated on a 7-point Likert-like scale ranging from strongly disagree to strongly agree for all items across the four domains. Since EC and VA are positively phrased and CB and VD 
are negatively phrased, we reverse scored the responses to the items for EC and VA so that higher scores represent more AFD problems in all four domains (i.e., greater communication difficulties and incongruence in values). The Rasch analysis revealed that the category thresholds did not increase monotonically with the categories, suggesting that the respondents were not clearly distinguishing all seven categories and the middle category was being used in an inconsistent manner. The analysis results suggested that the scale be rescored to represent four categories that were distinctly defined to represent ordered segments of the latent trait scale. Using Wright and Linacre's (1992) guidelines for rescoring, we refitted the Rasch model on the rescored data and produced thresholds that were now increasing monotinically. The rescored Categories 1-4 represented disagree, slightly disagree, slightly agree, and agree, with reverse scoring implemented for EC and VA. The Rasch-estimated AFD measures were initially centered on 0.0 with a range from $-\infty$ to $+\infty$, which is a true continuous interval on the logarithm of the odds scale that allows direct comparisons of the values to be made between youths and mothers within a domain. The centering on 0.0 was done for model identification purpose, a common approach in item response modeling. To increase generalizability of the measure to the entire family (youths, mothers, and fathers), we conducted Rasch analysis and centering using data from all respondents (i.e., including fathers). Because the structural equation model for this article was conducted only for mothers and youths, the centered means fluctuate but do not equal exactly 0.0 (see Table 1 for $M$ s and $S D$ s of the AFD domain scores for youths and mothers).

To interpret these means, one can compare them to the threshold locations for an average item difficulty level within a dimension. Thresholds distinguish two adjacent categories. The first threshold separates Category 1 from 2; the second, Category 2 from 3; and so forth. If a group's mean is less than the first threshold, then the group is, on average, in Category 1; if the mean falls between the first and second threshold, then the group is, on average, in Category 2; and so forth. The first, second, and third threshold estimates for an average item in the $\mathrm{CB}$ dimension are -2.77 , 0.67 , and 4.44, respectively. On average, youths $(M=0.37, S D=$ 1.96) and mothers $(M=-0.10, S D=2.42)$ are equivalent to Category 2 (slightly disagree), with youths reporting slightly higher communication problems than mothers. The threshold estimates for an average item in the EC dimension are 0.36, 2.70, and 5.88 , respectively. Thus, on average, youths $(M=0.52, S D=$ 2.22) are equivalent to Category 2 (slightly agree), and mothers $(M=-0.56, S D=2.58)$ are equivalent to Category 1 (agree), suggesting that since they are both on the agreement side of the spectrum, there is effective communication (since EC was reverse scored). For the remaining two dimensions, the first through third thresholds are $0.43,1.83$, and 4.76 for VA and $-1.77,0.24$, and 2.64 for VD. Youths reported slightly greater AFD problems on all four dimensions.

Depression. The Hamilton Depression Inventory (HDI) is a 23-item self-report inventory version of the Hamilton Depression Rating Scale, one of the most common measures of depression (Hamilton, 1960; Hamilton, 1967). There is strong support for the reliability and validity of the self-report HDI for assessment of the severity of depression in multiethnic samples (Dozois, 2003; Reynolds \& Kobak, 1995). The HDI evidenced good internal consistency $(\alpha=.93)$, test-retest reliability $(r=.95)$, and validity (content, criterion, and convergent) in its development study (Dozois, 2003; Reynolds \& Kobak, 1995). The HDI uses clinical cutoff scores for depression over the previous 2 weeks. A clinical cutoff score of 19 maximizes the hit rate (98.2\%), sensitivity $(99.3 \%)$, and specificity $(95.9 \%)$ in differentiation of nonreferred community adults from psychiatric outpatients diagnosed with major depression (Reynolds \& Kobak, 1995). The HDI evidenced good internal consistency in this study $(\alpha=.90)$, and the proportions of youths and mothers surpassing the HDI threshold score for

Table 1

Descriptive Statistics for Continuous Variables Used in the Structural Equation Model

\begin{tabular}{|c|c|c|c|c|c|}
\hline Variable & $M$ & $S D$ & Range & Skewness & Kurtosis \\
\hline 1. Youth age & 15.61 & 1.21 & $14-18$ & -0.044 & -1.222 \\
\hline 2. Mother age & 47.32 & 4.67 & $36-60$ & 0.349 & 0.081 \\
\hline 3. English language gap & 1.94 & 1.14 & -1 through 4 & -0.281 & -0.481 \\
\hline 4. Ethnic language gap & .93 & 1.34 & -3 through 4 & -0.073 & -0.219 \\
\hline 5. Heritage acculturation gap & 2.72 & 16.09 & -31 through 61 & 0.367 & 1.491 \\
\hline 6. Mainstream enculturation gap & 15.25 & 19.65 & -29 through 65 & 0.284 & -0.079 \\
\hline 7. AFD-Youth: EC & 0.52 & 2.22 & -4.58 through 5.87 & 0.204 & 0.003 \\
\hline 8. AFD-Youth: CB & 0.37 & 1.96 & -3.89 through 4.84 & 0.163 & -0.317 \\
\hline 9. AFD-Youth: VA & 0.31 & 1.45 & -3.10 through 4.15 & 0.302 & 0.261 \\
\hline 10. AFD-Youth: VD & 0.15 & 0.95 & -2.26 through 2.52 & -0.062 & -0.097 \\
\hline 11. AFD-Mother: EC & -0.56 & 2.58 & -5.31 through 6.97 & 0.330 & -0.151 \\
\hline 12. AFD-Mother: CB & -0.10 & 2.42 & -5.35 through 6.04 & -0.204 & -0.204 \\
\hline 13. AFD-Mother: VA & -0.33 & 1.63 & -3.29 through 4.62 & 0.102 & -0.487 \\
\hline 14. AFD-Mother: VD & -0.07 & 0.99 & -2.58 through 2.96 & -0.319 & 0.320 \\
\hline 15. Conflict-Youth & 13.56 & 3.95 & $6-24$ & 0.341 & -0.153 \\
\hline 16. Conflict-Mother & 11.09 & 4.02 & $6-21$ & 0.621 & -0.332 \\
\hline 17. HDI-Youth & 10.41 & 8.81 & $0.00-46.93$ & 1.460 & 2.556 \\
\hline 18. HDI-Mother & 7.84 & 6.85 & $0.00-37.90$ & 1.628 & 3.862 \\
\hline
\end{tabular}

Note. $\quad N$ ranges from 102 to 105 . The Acculturative Family Distancing (AFD) subscales are all scaled in the same direction, such that higher scores reflect greater communication difficulties and incongruence in values. Youths reported slightly higher AFD problems on all four dimensions $(\mathrm{EC}=\mathrm{Effective}$ Communication; $\mathrm{CB}=$ Communication Barriers; VA = Value Agreement; VD = Value Disagreement; HDI = Hamilton Depression Inventory). 
clinical depression were $15.3 \%$ (male $=13.5 \%$, female $=16.9 \%$ ) and $4.5 \%$, respectively.

Family conflict. The 36 -item Social Interactions Scale (SIS) measures positive and negative social interactions (family, friend, and spouse) on a 4-point Likert scale ranging from none at all to a lot (Kessler et al., 1994). The six items of the Family Conflict subscale focus on how family members argue, criticize, let each other down, and get on each other's nerves. The cross-cultural validity and reliability for the SIS among Chinese Americans have been previously confirmed (Hwang et al., 2000). Only the Family Conflict subscale was used in this study $(\alpha=.87)$, and higher scores represent higher conflict.

Language fluency gap. English and heritage language fluency were assessed with two items asking the participant how well they speak English and their ethnic or heritage language. Responses ranged from poor to excellent along a 5-point scale. We created a parent-child English gap score by subtracting parent scores from youth scores, such that higher scores represented a larger English gap, and we created a parent-child heritage language gap score by subtracting youth scores from parent scores, such that higher scores represented a greater gap.

Mainstream acculturation and heritage enculturation gap. The Vancouver Index of Acculturation (VIA) is a bidimensional measure of acculturation (Ryder, Alden, \& Paulhus, 2000). This 20-item measure provides two subscale scores for each participant, the degree of affiliation with his or her heritage culture and with the mainstream culture. Items on the VIA measure ethnic and mainstream behavior, participation, enjoyment, and social affiliation. Responses are answered on a 9-point Likert scale ranging from strongly disagree to strongly agree. This scale has been found to have strong internal consistency for the heritage $(\alpha=$ .79-.92) and mainstream ( $\alpha=.75-.89)$ dimensions, as well as strong concurrent validity with proxies of acculturation, including percentage of time residing in a Western country, percentage of time educated in the West, generational status, plans to remain in the West (vs. return to home country), English as a first language (vs. second language), and self-rated Western identification. Moreover, the measure also demonstrated good concurrent validity with scores on the Suinn-Lew Asian Self-Identity Acculturation Scale (Suinn et al., 1987), a unidimensional measure of acculturation. Coefficient alphas for affiliation to heritage and mainstream cultures in this study were .90 and .93 , respectively. In this study, the two dimensions of acculturation are differentiated and termed mainstream acculturation and heritage enculturation (Kim \& Omizo, 2006). We created parent-child mainstream acculturation gap scores by subtracting parent scores from youth scores, such that higher scores indicate greater acculturative difference, and we created parent-child heritage enculturation scores by subtracting youth scores from parent scores, such that higher scores are indicative of greater enculturative difference.

Place of birth and age. Participants reported their age and place of birth ( $0=$ U.S. born and $1=$ foreign born $)$.

\section{Data Analysis}

Structural equation modeling (SEM) with EQS 6.1 (Bentler, 2008) was used to test the primary study hypotheses; follow-up analyses on dichotomous depression dependent variables (DVs) were conducted in Mplus Version 5.2 (Muthén \& Muthén, 1998-
2007). EQS was chosen for the primary continuous-variable SEM analyses due to the availability of the Jamshidian-Bentler expectation maximization missing data procedure (Jamshidian \& Bentler, 1999), a maximum likelihood estimation approach for SEM models in which some data are missing, as well as availability of standard errors and fit indices robust to violations of distributional assumptions. However, for dichotomous analyses, EQS requires that measured variables are represented by their own latent variable, which was undesirable for this study because it added excessive free parameters to the model specification. Therefore, Mplus, which has no such requirements for its dichotomous variable models, was used for the dichotomous DV analysis; it features full information maximum likelihood imputation of missing data.

To address the first aim of the article - construct validity of the AFD measure-we compared a series of nested SEM measurement models using confirmatory factor analysis to determine the best fitting structure of the youth- and mother-reported AFD data, drawing on an SEM-based approach to multitrait multimethod matrix (MTMM) evaluation of discriminant and convergent validity (Byrne, 2006). Then, a correlation matrix-based MTMM approach was used to test the discriminant validity among AFD and a second family-level interaction construct: family conflict. Finally, we computed intraclass correlations to assess the degree of mother-youth agreement on the AFD subscales. To address the study's second aim, we fitted an SEM model using latent variables for AFD problems predicting youth and maternal depression via an intervening variable, family conflict. This model controls for potential confounds such as generational status, level of development (age), acculturation-enculturation gaps, and linguistic gaps. To assess mediation, we compared path coefficients in this model to those in a model in which the mediator was removed. Furthermore, to evaluate the plausibility of the directionality of effects implied in the meditational model, we also examined an alternative nested model in which youth and maternal AFD were the DVs in the model, predicted by depression and family conflict. Chi-square difference tests were used to evaluate comparative model fit. An alternate version of the primary SEM model with dichotomous depression outcomes in which youth and mothers were categorized as scoring over or under the HDI clinical cut-score was subsequently fit with Mplus. We evaluated the fourth and final aim of the study within the primary SEM model by assessing the path coefficients of generational status, level of development (age), and the acculturation-enculturation and linguistic gaps on the AFD latent variables.

\section{Results}

Table 1 presents the means, standard deviations, ranges, and values of skewness and kurtosis for all variables used for hypothesis testing. An intercorrelation matrix was prepared that included all study variables used for hypothesis testing (see Table 2). There were nine participants who had at least one variable missing (these participants each had between one and four variables missing; only two participants had as many as four missing variables).

Prior to analyzing data, we tested the assumption that all variables were normally distributed. Variables were standardized, and the $z$-score distributions were plotted. All had $z$ scores below absolute values of 3.5, suggesting no extreme outliers. Examina- 
Table 2

Intercorrelation Matrix for All Measured Variables in the Primary Structural Equation Model

\begin{tabular}{|c|c|c|c|c|c|c|c|c|c|c|c|c|c|c|c|c|c|c|}
\hline Variable & 2 & 3 & 4 & 5 & 6 & 7 & 8 & 9 & 10 & 11 & 12 & 13 & 14 & 15 & 16 & 17 & 18 & 19 \\
\hline 1. Youth age & .22 & -.02 & -.02 & .07 & .11 & .08 & .10 & .16 & .22 & .17 & .14 & .12 & .18 & .10 & -.07 & .05 & .16 & -.01 \\
\hline 2. Mother age & - & -.01 & .07 & .07 & .03 & .02 & -.15 & -.03 & -.01 & -.06 & -.08 & .02 & -.08 & -.02 & -.03 & -.07 & -.01 & -.04 \\
\hline 3. Place of birth & & - & -.04 & -.26 & .05 & .01 & .02 & -.05 & .14 & -.06 & .01 & .07 & .09 & .05 & .02 & -.15 & .03 & -.09 \\
\hline 4. English language gap & & & - & -.16 & .02 & .44 & .05 & -.07 & .01 & .03 & .16 & .24 & .15 & .21 & -.02 & -.03 & -.06 & .13 \\
\hline 5. Ethnic language gap & & & & - & .17 & .11 & .11 & .19 & .06 & .16 & .01 & -.02 & -.06 & -.03 & .05 & .14 & -.07 & -.15 \\
\hline 6. Heritage acculturation gap & & & & & - & -.02 & .37 & .32 & .39 & .42 & .21 & .19 & .29 & .22 & .19 & .08 & -.03 & .09 \\
\hline 7. Mainstream enculturation gap & & & & & & - & .02 & .11 & -.01 & .05 & .24 & .28 & .16 & .22 & .05 & -.05 & -.01 & -.05 \\
\hline 8. AFD-Youth: EC & & & & & & & - & .68 & .83 & .72 & .44 & .39 & .42 & .31 & .39 & .17 & .25 & .07 \\
\hline 9. AFD-Youth: CB & & & & & & & & - & .68 & 69 & .23 & .25 & .21 & .14 & .43 & .05 & .21 & -.08 \\
\hline 10. AFD-Youth: VA & & & & & & & & & - & .77 & .37 & .38 & .38 & .31 & .48 & .18 & .32 & .09 \\
\hline 11. AFD-Youth: VD & & & & & & & & & & - & .40 & .39 & .43 & .39 & .46 & .09 & .22 & -.03 \\
\hline 12. AFD-Mother: EC & & & & & & & & & & & - & .78 & .81 & .65 & .14 & .03 & .05 & .14 \\
\hline 13. AFD-Mother: CB & & & & & & & & & & & & - & .72 & .75 & .19 & -.02 & .02 & .16 \\
\hline 14. AFD-Mother: VA & & & & & & & & & & & & & - & .68 & .19 & .08 & .13 & .20 \\
\hline 15. AFD-Mother: VD & & & & & & & & & & & & & & - & .17 & .10 & .06 & .38 \\
\hline 16. Conflict-Youth & & & & & & & & & & & & & & & - & .13 & .35 & -.02 \\
\hline 17. Conflict-Mother & & & & & & & & & & & & & & & & - & .23 & .44 \\
\hline 18. HDI-Youth & & & & & & & & & & & & & & & & & - & .14 \\
\hline 19. HDI-Mother & & & & & & & & & & & & & & & & & & - \\
\hline
\end{tabular}

Note. $\quad N$ ranges from 101 to $105 . r$ s $<-.19$ and $>.19^{*}$ are $p<.05$, two-tailed. The Acculturative Family Distancing (AFD) subscales are all scaled in the same direction, such that higher scores reflect greater communication difficulties and incongruence in values. Youths reported slightly higher AFD problems on all four dimensions. EC = Effective Communication; $\mathrm{CB}=$ Communication Barriers; VA = Value Agreement; VD = Value Disagreement; HDI = Hamilton Depression Inventory

tion of the raw score plots and frequencies suggested approximately normal distributions, with modest skewness or kurtosis in four of 19 variables (mother and youth HDI scores, heritage gap, and youth age) although in no case exceeding the values of 2 and 7 , respectively, which were suggested by West, Finch, and Curran (1995) as indicators of violations of distributional assumptions (see Table 1). Multivariate tests of distributional assumptions were also within acceptable limits. Robust maximum likelihood estimation, which generates fit indices and standard errors that are correct even when distributional assumptions are violated, was used to confirm model fit.

\section{Convergent and Discriminant Validity of the AFD Measure}

Drawing on an SEM-based MTMM approach to assess convergent and discriminant validity (Byrne, 2006), we compared a null model (in which all four youth-reported and all four motherreported AFD subscales loaded on one latent factor) with a traitonly model (one latent variable for the four youth- and motherreported communication subscales, and one for the four youth- and mother-reported values subscales), a method-only model (one latent variable for the four youth-reported subscales, and one for the four mother-reported subscales), and a method-and-trait model (two latent variables corresponding to the communication and values domains, and two corresponding to the mother- and youthreported method variance). Each model fit the data better than the last, such that the method-and-trait model, $\chi^{2}(10)=12.18, p=$ $.27>$ the method-only model, $\chi^{2}(19)=39.24, p<.01>$ the trait-only model, $\chi^{2}(19)=269.22, p<.00001>$ null model, $\chi^{2}(20)=220.22, p<.00001$. The superior fit of the method-andtrait model as compared with the method-only model, $\chi_{\text {diff }}^{2}(9)=$ 27.06, $p<.01$, suggests that the AFD trait scores are influenced by a common perception shared among youths and mothers, and not just method variance, supporting convergent validity. In the method-only model, the correlation between the mother- and youth-reported latent variables was statistically significant and of moderate to large magnitude $(r=.48, p<.01)$, offering additional support for the convergent validity of the measure. The methodand-trait model was also compared with a similar model with factors for both methods but only one (unidimensional) AFD trait that did not distinguish between communication and values. The model distinguishing between these two dimensions led to better model fit, $\chi_{\text {diff }}^{2}(2)=18.79, p<.001$, offering evidence of the discriminant validity of the subscales produced by the Rasch analysis (see Method section). The fit indices (comparative fit index [CFI], root-mean-square error of approximation [RMSEA]) for the method-and-trait model were .997 and .045 , and for the method-only model were .969 and .10. For hypothesis testing in the structural model (to address the second and third study aims), the method-only measurement model was adopted. The methodonly model was used because, in the structural model, the total number of estimated parameters relative to the $N$ of 105 was high, given the number of control variables and free paths. By adopting the method-only model, we reduced the number of total parameters estimated while still maintaining a representation of AFD with an excellent CFI. Modeled this way, the AFD factors represent the common variance shared among the four subscales, presumably representing the underlying AFD trait that manifests in a variety of specific ways.

We examined convergent and discriminant validity at the level of the correlation matrix (see Table 2) using an MTMM perspective, with a focus on the AFD subscales and the family conflict subscales (each rated by mothers and youth). There were moderate correlations among the two informants on corresponding AFD 
subscales, offering evidence of convergent validity, while crossinformant correlations between AFD and family conflict were weak and nonsignificant, providing evidence of discriminant validity. To further assess youth-mother agreement on the specific subscales, we computed intraclass correlation coefficients (ICC, two-way random average measures, consistency) for the four AFD subscales: $\mathrm{EC}(.61, p<.0001), \mathrm{CB}(.39, p<.01)$, VA $(.55, p<$ $.0001)$, and VD $(.56, p<.0001)$. These ICCs reflect moderate mother-child agreement, which we accounted for in the structural models discussed later by freeing the correlation between mother and child AFD latent variable scores. Finally, the association between the measures of the acculturation gap and the AFD subscales was examined, which showed that these measures were well discriminated from one another, while sharing some common variance (see Table 2). In sum, there was support for the construct validity of the refined AFD measure.

\section{Comparative Structural Models}

As for the second study aim and its corresponding hypothesis, Figure 1 presents the estimated structural model for youth and mother depressive symptoms (with standardized path coefficients). In addition to the predicted paths, all variances of measured variables and factors were allowed to vary freely. In order to control for the seven covariates (youth's place of birth, youth's age, mother's age, English language gap score, heritage language gap score, mainstream acculturation gap score, and heritage enculturation gap score), we set these variables as predictors of the AFD, family conflict, and depression variables and also allowed them to intercorrelate freely among themselves in the specified model. For the sake of clarity, only significant paths linking these covariates with the other variables are depicted in Figure 1 . The model fit the data well, $\chi^{2}(100, N=105)=130.31, p=.02$;
$\mathrm{CFI}=.964 ; \mathrm{RMSEA}=.052 ; 90 \%$ confidence intervals $(\mathrm{CI})[.017$, .076]. When robust fit indices were used, comparable parameters were obtained: Yuan-Bentler scaled $\chi^{2}(100, N=105)=118.61$, $p=.09$; robust $\mathrm{CFI}=.973$; robust $\mathrm{RMSEA}=.041 ; 90 \% \mathrm{CIs}[.00$ $.068]$.

In the SEM model (Figure 1), even after controlling for the seven covariates, we found that more youth-reported AFD was associated with greater youth-reported family conflict. In turn, higher levels of both youth-reported and mother-reported family conflict predicted more youth depressive symptoms. In contrast, neither AFD factor was associated with mother-reported family conflict; instead, mother-reported AFD had a significant direct association with maternal depressive symptoms. Indirect effects of the hypothesized paths were also examined (not depicted in Figure 1). The heritage enculturation gap had an indirect effect on youthreported family conflict via the AFD latent variables (Sobel test: $t=3.30, p<.01$ ), such that a larger gap was associated with more conflict. The youth AFD factor had an indirect effect on youthreported depression via family conflict, with more AFD problems predicting more depressive symptoms, $t=2.56, p<.05$.

Mediation. To test for mediation, we fitted a model omitting the family conflict variables. Model fit was relatively good, $\chi^{2}(87)=123.39, p<.01 ;$ CFI $=.952$; RMSEA $=.062$. The direct paths from mother-reported AFD to maternal depressive symptoms and from youth-reported AFD to youth depressive symptoms were .25 and .41 ( $p \mathrm{~s}<.05)$, respectively. The comparable path coefficients in the model that included family conflict as a mediator (in Figure 1) were $.27(p<.05)$ and $.23(n s)$, respectively. The reduction of the direct path from youth-reported AFD to youth depression to a nonsignificant effect in the mediated model is consistent with partial mediation. This conclusion is supported by the significant indirect effect from youth-reported

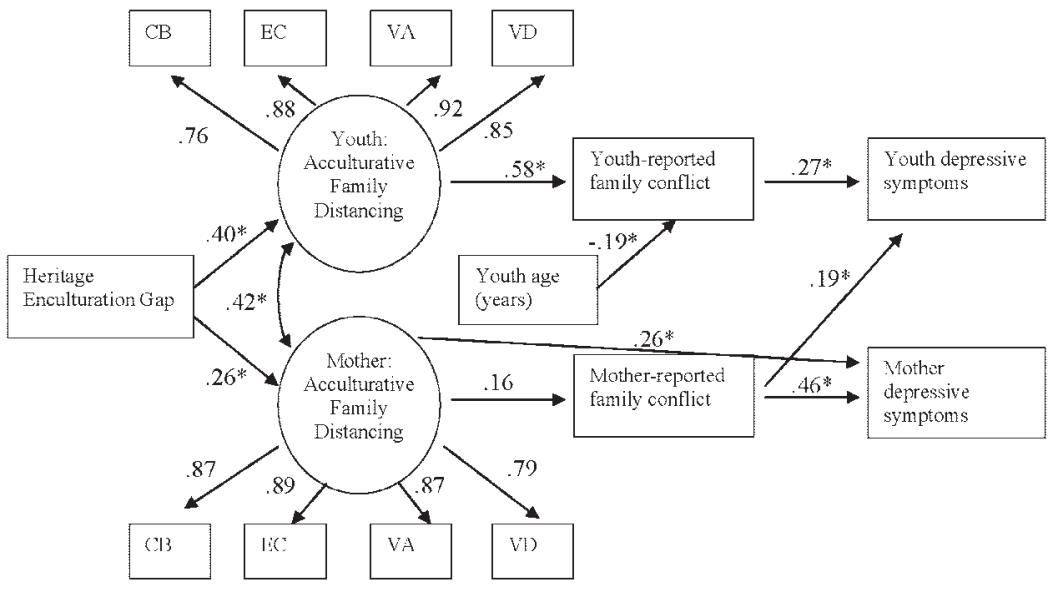

Figure 1. Structural equation model for the acculturative family distancing (AFD) model. All values are standardized path coefficients. Covariates in the model are place of birth, youth age, mother age, English language gap score, heritage language gap score, mainstream acculturation gap score, and heritage enculturation gap score. Nonsignificant covariate paths are not depicted. The direct paths from youth AFD to youth depressive symptoms and from youth conflict to maternal depressive symptoms are nonsignificant and therefore not depicted. All latent variable loadings are statistically significant $(p<.05)$. AFD subscales are EC $=$ Effective Communication; $\mathrm{CB}=$ Communication Barriers; VA = Value Agreement; VD = Value Disagreement. The AFD scales are all scaled in the same direction, such that higher scores reflect greater communication difficulties and incongruence in values. ${ }^{*} p<.05$. 
AFD to youth depressive symptoms via family conflict, reported earlier. In contrast, there was no evidence of a mediated effect from AFD to maternal depression.

Alternative ordering of variables in the model. A model nested within the primary SEM model presented in Figure 1 was evaluated, in which youth and mother depression and family conflict variables were predictors of the two AFD latent variables, which were intercorrelated. The same seven covariates were retained in the model, predicting all other variables. Model fit was borderline, $\chi^{2}(128)=198.14, p<.0001, \mathrm{CFI}=.912, \mathrm{RMSEA}=$ .071 , and the chi-square difference test indicated that the representation of the covariance structure modeled in Figure 1 was a better fit to the data, $\chi_{\text {diff }}^{2}(28)=67.83, p<.001$. Therefore, the model in which AFD predicted family conflict, which predicted depression, was retained as the better fitting model.

\section{Exploratory Categorical Analyses}

As noted in the Method section, $15.3 \%$ of youths and $4.5 \%$ of the mothers scored above the HDI threshold score for clinical depression. Variability in this DV is limited, thus rendering analyses with a relatively modest sample size exploratory in scope. We fit an SEM model using clinical depression as the DV $(0=$ not depressed, 1 = depressed), drawing from the results of the results of the primary SEM model depicted in Figure 1. To reduce the number of variables and free parameters in the model, given the limited variability in the DV, we dropped the five covariates that had no significant path coefficients in the primary model, and we restricted the two other covariates to freely predict only the variables for which significant path coefficients had emerged in the primary model (heritage enculturation to the AFD latent variables, and youth age to youth-reported conflict). Model fit was good, $\chi^{2}(20)=25.21, p=.19, \mathrm{CFI}=.950$, RMSEA $=.050$. All significant path coefficients obtained in the primary SEM model (Figure 1) were also found significant in the dichotomous model. There was also a significant indirect effect from youth-reported AFD to clinical depression via the family conflict variables (Sobel test: $t=2.57, p<.05$ ); the indirect effect for mother's depression was nonsignificant. Hence, AFD and family conflict are likely relevant to the prediction of clinical depression in Asian American youth and their mothers.

\section{Predictors of AFD}

The seven covariates included in the primary SEM analyses were putative predictors of AFD. Of these variables, only the heritage enculturation gap was a significant predictor of the mother- and youth-reported AFD latent variables (see Figure 1). For both mothers and youths, a greater heritage enculturation gap among mothers and children was associated with more AFDrelated problems. In the model, generational status, the language gap scores, the acculturation gap, and youth and maternal age were not significant simultaneous predictors of AFD.

\section{Discussion}

In this study, the theory and construct of AFD (Hwang, 2006a) were tested in a sample of high school youths and mothers. AFD increased the risk for youth depression, partially mediated by family conflict. In this study, we expanded upon earlier research (Hwang \& Wood, 2009) by testing the AFD model on a specific Asian American group (i.e., Chinese Americans); focusing on youths still living at home with their parents, who would experience the effects of AFD more regularly than college students living away from home (cf. Hwang \& Wood, 2009); utilizing two informants (mother and youth) rather than exclusively youth selfreport; refining AFD measurement through Rasch modeling; using an MTMM approach to assess convergent and discriminant validity; evaluating the influences of the mainstream acculturation and heritage enculturation gap, heritage and mainstream linguistic gap, age, and place of birth on AFD as well as the other variables in the models; and testing the model on both youth and mother outcomes.

Rasch analysis resulted in a refined AFD instrument with a reconfigured response category scheme and a more accurate dimensional structure. The measure was reduced from 46 items to 29 items, and the item-response categories were reduced from a 7-point scale to a 4-point scale because respondents were not clearly distinguishing among all seven response categories and the middle category was not being used in a consistent manner. A four-dimensional structure composed of two subdimensions for each of the AFD domains was identified and found to best fit the data and the AFD construct (i.e., for communication-EC and $\mathrm{CB}$ subscales; for values-VA and VD subscales). A MTMM matrix approach was taken to analyze convergent and discriminant validity. The observed pattern of correlations and comparative SEM models was supportive of moderate convergence among different informants on AFD, as well as clear discrimination between AFD and family conflict and between the communication and values dimensions, meeting classical criteria for construct validity within the MTMM context. Notably, youth-mother agreement on AFD was much stronger than youth-mother agreement on family conflict (measured by a well-validated, commonly used scale), a finding underscoring the potential sensitivity of the AFD instrument. Moreover, AFD and the acculturation-enculturation gap shared some common variance but were well discriminated. All told, there was considerable support for the construct validity of the refined AFD measure.

With regard to predictors of AFD, results indicated that larger mother-youth heritage enculturation gaps were associated with greater mother- and youth-reported AFD problems. This suggests that the difference in enculturation level between mothers who retain their culture of origin and youths who either never acquired or lost part of their heritage culture over time puts families at risk for developing AFD and subsequent family problems. The retention of heritage culture may serve as a culturally protective factor and help improve family relations and youth mental health. The heritage enculturation gap also had an indirect effect on family conflict for youths, suggesting that the enculturation gap may increase AFD-related difficulties in families and trigger family conflict. The enculturation gap has previously been found to affect child maladjustment in one study on Chinese Americans (Costigan \& Dokis, 2006). It is possible that the lack of association between the mainstream acculturation gap and family or individual adjustment problems in this study, and the greater importance of the heritage enculturation gap, may be due to traditional acculturation measures being more sensitive for detection of differences in enculturation gaps than in acculturation gaps. However, it may also be that youths are more likely to accept that their parents are 
unlikely to fully acculturate, whereas, parents are less accepting that their children do not acquire and retain their heritage culture, a commonly noted point of disagreement (Kim \& Omizo, 2006). The notion that enculturation gaps are more influential than acculturation gaps has also been reported in one study of Latino family process (Smokowski, Rose, \& Bacallao., 2008) and one study of Muslim American student depression (Asvat \& Malcarne, 2008). In the future, researchers should focus on further understanding of the types of acculturation-enculturation gaps that are responsible for family dysfunction and AFD. For example, Asvat and Malcarne (2008) found that regardless of an individual's enculturation level, it was personal and family mismatch in enculturation that was associated with depressive symptoms.

It was surprising to find that both mainstream and heritage language gaps did little to influence the overall model. This finding runs contrary to the expectation that differential language fluencies harm mother-youth communication (Liu et al., 2009; Tseng \& Fuligni, 2000; Weaver \& Kim, 2008). It may be that the language gap measure, which consisted of mother and youth single-item self-reports of their own language fluency, was insufficient to measure this complex phenomenon. Since few multi-item language fluency measures have been developed to facilitate psychological research, researchers should focus on developing more suitable instruments. For example, separate subscores could be developed for speaking, reading, and writing. An oral assessment instrument that measures not only self-reported fluency but also the ability to get one's point across effectively, comfort in expressing oneself, listening comprehension, and ability to discuss feelings as well as concrete needs may also be beneficial.

In this study, we built on AFD research by examining both mother and youth reports of AFD, family conflict, and depression as a symptomatic and diagnostic outcome. Despite the relatively small sample size, both outcome models (i.e., continuous and categorical representations of depression) yielded comparable results. Overall, greater youth and mother reports of AFD were associated with higher depressive symptoms and risk for clinical depression. These findings remained robust even after more general acculturation-gap phenomena (i.e., mainstream acculturation and heritage enculturation gaps and linguistic gaps) were controlled. Family conflict partially mediated this relation for youth depression. The inclusion of mothers makes a unique contribution because most studies of the acculturation gap have focused on youth outcomes. It is unclear why mothers' reports of family conflict did not mediate the relation between mother-reported AFD and maternal depression as it did for youths. Instead, mothers' reports of AFD directly increased risk for mothers' depression. It may be that mothers underreported their distress level and family problems, perhaps due to stigma about talking about family problems and mental illness evident in Chinese culture (Hwang et al., 2000). This is supported by trends found in the data. Specifically, mothers reported lower overall depressive symptoms, family conflict, and AFD and evidenced lower rates of clinical depression on the HDI than youths did. However, depression is also typically higher in late adolescents and early adulthood (Hwang et al., 2005).

Another possibility is that mothers and youths have different attributions as to why they argue. Youths may perceive that AFD (not being able to communicate effectively and having incongruent cultural values) is the primary reason that they fight with their parents. Mothers may attribute conflict to other reasons (e.g. youth disobedience, lack of filial piety), and instead, perceive AFD as a reason that they are depressed (i.e., "My children don't talk to me"; "My children have lost their cultural heritage"), especially given the many sacrifices that immigrant parents make for their children. It is also possible that because the Family Conflict subscale is a broader measure of family process than the AFD measure (i.e., it measures family conflict rather than mother-child conflict), parents may have reported differently than did youths. For example, when youths think about family conflict, they may think about conflict with their parents. However, when mothers think about family conflict, they may think about conflict with children as well as with their spouse. Similarly, on the AFD measure, youths may respond thinking about their parents as a unit, while parents may respond thinking about their relationship with a single target child in the family, raising the possibility that youth-reported AFD is more homologous with general family conflict than is mother-reported AFD. Differences between parents and children in perceptions and attributions to individual and family problems should be explored in future research.

Understanding how emic (culture-specific) and etic (cultureuniversal) factors interplay and contribute to mental health problems (i.e., how etic phenomena such as family conflict can mediate the effects of emic phenomena such as AFD processes in increasing risk for psychopathology) is important because it helps psychologists understand problem development across cultures and in immigrant cultures that are in transition. For example, even though some degree of family conflict may be normative in all cultures, culture-specific factors such as AFD may not affect Chinese families in Asia or European American families in the United States because they are not immigrants in transition. Results from this study also help in the integration of a disparate set of research on acculturation, family conflict, and mental health outcomes. Given the high prevalence of family conflict in Asian American families (Greenberger \& Chen, 1996; Lee \& Liu, 2001; Lee, Su, \& Yoshida, 2005) and high psychological distress evident in Asian American youths (Abe \& Zane, 1990; Cheng, Leong, \& Geist, 1993; Greenberger \& Chen, 1996; Okazaki, 1997), more research needs to be conducted in this arena. Intergenerational conflict and the cultural divide between generations (e.g., social isolation, loss of filial piety, expectations for taking care of aging parents) has been found to increase risk for depression and suicide among Asian American elderly (Diego, Yamamoto, Nguyen, \& Hifumi, 1994), who as a group proportionally evidence the highest rate of suicide in the United States (McKenzie, Serfaty, \& Crawford, 2003).

In this study, we utilized a novel approach to examine acculturative issues in psychosocial problem development for Chinese American families. However, there are a number of limitations that deserve attention and that moderate the conclusions that can be drawn from this study. First, data were cross sectional. Longitudinal studies should be conducted to assess issues of timing and sequencing and the direction of effects. In future studies, researchers should examine how AFD processes affect family relations over time and developmental periods (e.g., tracking families from primary school through later adulthood). Second, data were collected on a subsample of high school students at a single school. Findings may not generalize to other age groups, settings, or other students at the same school. Although it was informative to have 
mother reports of AFD and conflict, understanding AFD within the full family context (i.e., inclusion of father reports) would have also been useful; unfortunately, a sufficiently representative sample of father reports was not available in this study. Third, the participants in this study completed a 46-item version that was later reduced to a 29-item form. This leaves open the possibility that a different ordering of items may have affected results had the final version of the measure been used initially. Fourth, the method-only measurement model for AFD selected for the structural model had one fit index that suggested adequate fit and a second that was borderline. Kline (2005) has noted the need to consider not only model fit but also what makes sense in the selection of final SEM models, and in this case, the relatively small difference in fit (compared with the method-and-trait model) plus the preference for parsimony in the structural model made the method-only model the most sensible option. The final structural models fit adequately with this representation of AFD. Fifth, a larger sample size would also allow for inclusion of more variables and complex modeling (e.g., tests of moderation). Since not all families with an acculturation gap develop difficulties, other important intervening variables that increase or reduce risk (e.g., personality factors, the role of extended family) also should be explored.

Finally, data were collected via self-report methodology, which has both advantages and disadvantages. One advantage of selfreports is that participants may feel less embarrassed to address sensitive issues (which may be even more important for cultural groups that value privacy) and may be more likely to provide honest assessments of stigmatizing topics. On the other hand, face-to-face interviews may reduce confusion and provide participants the opportunity to ask questions and clarify responses, and direct observations can also overcome some forms of respondent bias. Relatedly, use of self-report measures introduces the possibility of method variance affecting the results. Although there were several cross-informant findings in the structural models (e.g., the link between mother-reported conflict and youth depression), many of the findings were intrainformant, which can be influenced by method variance. Self-reports can also be biased, and different respondents may use of different frames of reference when answering questions, thus potentially influencing the accuracy of difference scores (e.g., mothers may use other immigrant mothers as a frame of reference when reporting their language fluency, and youths may use English-fluent Americans). More research should be conducted on development of a more reliable and valid measure of linguistic fluency. In future studies, investigators should also evaluate the best methods for examining "gap" phenomena (e.g., acculturation-enculturation and heritage-mainstream language gaps). For example, are difference scores the best method for accurately assessment of parent-youth gaps, and what is the relationship between component scores and difference scores?

The results of this study suggest that a theoretically based conceptualization of a more proximally defined acculturation-gap phenomena (i.e., AFD-breakdowns in communication and disruptive discrepancies in cultural values) may provide clarification of the linkages among acculturation processes, family problems, and mental health outcomes. Although nearly all immigrant families evidence some form of acculturation gap, not all immigrant families develop problems. The broad and general definition of the acculturation gap may be insufficient for the identification of distinct mechanisms of risk. Our use in this study of AFD rather than more distal proxies of the acculturation gap may help us to identify specific foci for intervention (see Hwang, 2006a, for clinical illustrations). For example, programs that target improving parent-youth communication difficulties may help decrease family conflict and subsequent parent and youth depression. The lack of a significant effect of linguistic gaps on the primary outcome variables indicates that focus of such communication training might need to be broader than improvement of language fluency. Communication difficulties can also be influenced by cultural differences in communication styles (e.g., the degree of directness vs. indirectness or verbal language vs. nonverbal body and facial language), which may also need to be addressed through psychoeduction (Sue, 1990). Results from this study also indicate that it may be particularly important for youths to learn more about their heritage culture and find ways of integrating it with their adoption of mainstream culture to avoid exacerbating AFD and indirectly magnifying family conflict. Although two programs have been developed to improve immigrant family relations (one for Chinese Americans and one for Hispanic families; Szapocznik, Santisteban, Kurtines, Perez-Vidal, \& Hervis, 1984; Ying, 1999), more should be done to address proximal mechanisms of risk for depression and other mental health problems.

\section{References}

Abe, J. S., \& Zane, N. W. (1990). Psychological maladjustment among Asian and White American college students: Controlling for confounds. Journal of Counseling Psychology, 37, 437-444.

Adams, R. J., Wilson, M., \& Wang, W.-C. (1997). The multidimensional random coefficients multinomial logit model. Applied Psychological Measurement, 21, 1-23.

Andrich, D. (1978). A rating formulation for ordered response categories. Psychometrika, 43, 561-573.

Andrich, D. (1996). Measurement criteria for choosing among models with graded responses. In A. von Eye \& C. C. Clogg (Eds.), Categorical variables in developmental research: Methods of analysis (pp. 3-35). San Diego, CA: Academic Press.

Asvat, Y., \& Malcarne, V. L. (2008). Acculturation and depressive symptoms in Muslim university students: Personal-family acculturation match. International Journal of Psychology, 43, 114-124.

Bentler, P. M. (2008). EQS: A structural equation program (Version 6.1) [Computer software]. Encino, CA: Multivariate Software.

Berry, J. W. (2003). Conceptual approaches to acculturation. In K. M. Chun, P. B. Organista, and G. Marin (Eds.), Acculturation: Advances in theory, measurement, and applied research (pp. 163-186). Washington DC: American Psychological Association.

Birman, D. (2006). Measurement of the "acculturation gap" in immigrant families and implications for parent-child relationships. In M. H. Bornstein and L. R. Cote (Eds.), Acculturation and parent-child relationships: Measurement and development (pp. 113-134). Mahwah, NJ: Erlbaum.

Bornstein, M. H., \& Cote, L. R. (2006). Acculturation and parent-child relationships: Measurement and development. Mahwah, NJ: Erlbaum.

Byrne, B. M. (2006). Structural equation modeling with EQS: Basic concepts, applications, and programming. Mahwah, NJ: Erlbaum.

Cheng, D., Leong, F. T., \& Geist, R. (1993). Cultural differences in psychological distress between Asian and Caucasian American college students. Journal of Multicultural Counseling and Development, 21, 182-190.

Chung, R. (2001). Gender, ethnicity, and acculturation in intergenerational conflict of Asian American college students. Cultural Diversity and Ethnic Minority Psychology, 7, 376-386. 
Costigan, C. L., \& Dokis, D. P. (2006). Relations between parent-child acculturation differences and adjustment within immigrant Chinese families. Child Development, 77, 1252-1267.

Crane, D. R., Ngai, S. W., Larson, J. H., \& Hafen, M. A. (2005). The influence of family functioning and parent-adolescent acculturation on North American Chinese adolescent outcomes. Family Relations, 54, $400-410$

Diego, A. T., Yamamoto, J., Nguyen, L. H., \& Hifumi, S. S. (1994). Suicide in the elderly: Profiles of Asians and Whites. Asian American and Pacific Islander Journal of Health, 2, 49-57.

Dinh, K. T., \& Nguyen, H. H. (2006). The effects of acculturative variables on Asian American parent-child relationships. Journal of Social and Personal Relationships, 23, 407-426.

Dozois, D. J. A. (2003). The psychometric characteristics of the Hamilton Depression Inventory. Journal of Personality Assessment, 80, 31-40.

Embretson, S. E., \& Reise, S. P. (2000). Item response theory for psychologists: Mahwah, NJ: Erlbaum.

Escobar, J. I., \& Vega, W. A. (2000). Mental health and immigration's AAA: Where are we and where do we go from here? Journal of Nervous and Mental Diseases, 188, 736-740.

Farver, J. A., Narang, S. K., \& Bhadha, B. R. (2002). East meets West: Ethnic identity, acculturation, and conflict in Asian Indian families. Journal of Family Psychology, 16, 338-350.

Fujimoto, K., Hwang, W., \& Wood, J. J. (2010). Acculturative family distancing: Evaluation of the psychometric properties through Rasch modeling. Manuscript in preparation.

Gil, A. G., \& Vega, W. A. (1996). Two different worlds: Acculturation stress and adaptation among Cuban and Nicaraguan families. Journal of Social and Personal Relationships, 13, 435-456.

Greenberger, E., \& Chen, C. (1996). Perceived family relationships and depressed mood in early and late adolescence: A comparison of European and Asian Americans. Developmental Psychology, 32, 707-716.

Hamilton, M. (1960). A rating scale for depression. Journal of Neurology, Neurosurgery, and Psychiatry, 23, 56-62.

Hamilton, M. (1967). Development of a rating scale for primary depressive illness. The British Journal of Social and Clinical Psychology, 6, $278-$ 296.

Hwang, W. (2006a). Acculturative family distancing: Theory, research, and clinical practice. Psychotherapy: Theory, Research, Practice, Training, 43, 397-409.

Hwang, W. (2006b). Acculturative Family Distancing Measure: Parent $(P R)$ and youth (YR) reports (English, Chinese, and Spanish versions). Unpublished copyrighted questionnaire.

Hwang, W., Chun, C., Takeuchi, D. T., Myers, H. F., \& Prabha, S. (2005). Age of first-onset major depression in Chinese Americans. Cultural Diversity and Ethnic Minority Psychology, 11, 16-27.

Hwang, W., Myers, H. F., \& Takeuchi, D. T. (2000). Psychosocial predictors of first-onset depression in Chinese Americans. Social Psychiatry Psychiatric Epidemiology, 35, 133-145.

Hwang, W., \& Wood, J. J. (2009). Acculturative family distancing (AFD) in immigrant families: A structural model of linkages with mental health outcomes among young adults. Child Psychiatry and Human Development, 40, 123-138.

Jamshidian, M., \& Bentler, P. M. (1999). ML estimation of mean and covariance structures with missing data using complete data routines. Journal of Educational and Behavioral Statistics, 24, 21-24.

Kessler, R. C., McGonagle, K. A., Zhao, S. N., Nelson, C. B., Hughes, M., Eshleman, S., .. . Kendler, K. S. (1994). Lifetime and 12-month prevalence of $D S M-I I I-R$ psychiatric disorders in the United States: Results from the National Comorbidity Study. Archives of General Psychiatry, $51,8-19$

Kim, B. S., \& Omizo, M. M. (2006). Behavioral acculturation and enculturation and psychological functioning among Asian American college students. Cultural Diversity and Ethnic Minority Psychology, 12, 245258.

Kim, S. Y., Chen, Q., Li, J., Huang, X., \& Moon, U. J. (2009). Parent-child acculturation, parenting, and adolescent depressive symptoms in Chinese immigrant families. Journal of Family Psychology, 23, 426-437.

Kline, R. (2005). Principles and practice of structural equation modeling. New York: Guilford Press.

Kwak, K. (2003). Adolescents and their parents: A review of intergenerational family relations for immigrants and non-immigrant families. $\mathrm{Hu}$ man Development, 46, 115-136.

Larsen, L. J. (2004). The foreign-born population in the United States. 2003. Washington, DC: U.S. Census Bureau.

Lee, R. M., Choe, J., Kim, G., \& Ngo, V. (2000). Construction of the Asian American family conflicts scale. Journal of Counseling Psychology, 47, 211-222.

Lee, R. M., \& Liu, H. T. T. (2001). Coping with intergenerational family conflict: Comparison of Asian American, Hispanic, and European American college students. Journal of Counseling Psychology, 48, 410419.

Lee, R. M., Su, J., \& Yoshida, E. (2005). Coping with intergenerational family conflict among Asian American college students. Journal of Counseling Psychology, 52, 389-399.

Lim, S., Yeh, M., Liang, J., Lau, A. S., \& McCabe, K. (2009). Acculturation gap, intergenerational conflict, parenting style, and youth distress in immigrant Chinese American families. Marriage \& Family Review, $45,84-106$.

Liu, L. L., Benner, A. D., Lau, A. S., \& Kim, S. Y. (2009). Motheradolescent language proficiency and adolescent academic and emotional adjustment among Chinese American families. Journal of Youth and Adolescence, 38, 572-586.

McGoldrick, M., Giordano, J., \& Garcia-Preto, N. (2005). Ethnicity and family therapy (3rd ed.). New York, NY: Guilford Press.

McKenzie, K., Serfaty, M., \& Crawford, M. (2003). Suicide in ethnic minority groups. British Journal of Psychiatry, 183, 100-101.

Muthén, L. K., \& Muthén, B. O. (1998-2007). Mplus user's guide. Los Angeles, CA: Authors.

Okazaki, S. (1997). Sources of ethnic differences between Asian American and White American college students on measures of depression and social anxiety. Journal of Abnormal Psychology, 106, 52-60.

Reynolds, W. M., \& Kobak, K. A. (1995). Hamilton Depression Inventory (HDI): A self-report version of the Hamilton Depression Rating Scale (professional manual). Odessa, FL: Psychological Assessment Resources.

Rogler, L. H., Cortes, D. E., \& Malgady, R. G. (1991). Acculturation and mental health among Hispanics: Convergence and new directions for research. American Psychologist, 46, 585-597.

Rosenthal, D., Ranieri, N., \& Klimidis, S. (1996). Vietnamese adolescents in Australia: Relationships between perceptions of self and parental values, intergenerational conflict, and gender dissatisfaction. International Journal of Psychology, 31, 81-91.

Ryder, A. G., Alden, L. E., \& Paulhus, D. L. (2000). Is acculturation unidimensional or bidimensional? A head-to-head comparison in the prediction of personality, self-identity, and adjustment. Journal of Personality \& Social Psychology, 79, 49-65.

Sluzki, C. (1979). Migration and family conflict. Family Process, 18, 379-390.

Smokowski, P. R., Rose, R., \& Bacallao, M. L. (2008). Acculturation and Latino family processes: How cultural involvement, biculturalism, and acculturation gaps influence family dynamics. Family Relations, 57, 295-308.

Su, J., Lee, R. M., \& Vang, S. (2005). Intergenerational family conflict and coping among Hmong American college students. Journal of Counseling Psychology, 52, 482-489.

Sue, D. W. (1990). Culture-specific strategies in counseling: A conceptual 
framework. Professional Psychology: Research and Practice, 21, 424433.

Suinn, R. M., Rickard-Figueroa, K., Lew, S., \& Vigil, P. (1987). The Suinn-Lew Asian Self-Identity Acculturation Scale: An initial report. Educational and Psychological Measurement, 47, 401-407.

Szapocznik, J., \& Kurtines, W. M. (1993). Family psychology and cultural diversity: Opportunities for theory, research, and application. American Psychologist, 48, 400-407.

Szapocznik, J., Santisteban, D., Kurtines, W., Perez-Vidal, A., \& Hervis, O. (1984). Bicultural effectiveness training: A treatment intervention for enhancing intercultural adjustment in Cuban American families. Hispanic Journal of Behavioral Sciences, 6, 317-344.

Tseng, V., \& Fuligni, A. J. (2000). Parent-adolescent language use and relationships among immigrant families with East Asian, Filipino, and Latin American backgrounds. Journal of Marriage and the Family, 62, 465-476.

U.S. Census Bureau. (2002). United States census: 2002 [Data file]. Available from http://www.census.gov

Vega, W. A., Khoury, E. L., Zimmerman, R. S., \& Warheit, G. J. (1995). Cultural conflicts and problem behaviors of Latino adolescents in home and school environments. Journal of Community Psychology, 23, 167179.

Weaver, S. R., \& Kim, S. Y. (2008). A person-centered approach to studying the linkages among parent-child differences in cultural orientation, supportive parenting, and adolescent depressive symptoms in
Chinese American families. Journal of Youth and Adolescence, 37, $36-49$.

West, S. G., Finch, J. F., \& Curran, P. J. (1995). Structural equation models with non-normal variables: Problems and remedies. In R. H. Hoyle (Ed.), Structural equation modeling (pp. 56-75). Thousand Oaks, CA: Sage.

Wilson, M. (2005). Constructing measures: An item response modeling approach: Mahwah, NJ: Erlbaum.

Wolfe, E. W., \& Smith, E. V., Jr. (2007). Instrument development tools and activities for measure validation using Rasch models: Part II. Validation activities. In E. V. Smith, Jr., \& R. M. Smith (Eds.), Rasch measurement: Advanced and specialized applications (pp. 243-290). Maple Grove, MN: Journal of Applied Management Press.

Wright, B. D., \& Linacre, J. M. (1992). Combining and splitting categories. Rasch Measurement Transactions, 6, 233-235.

Wu, M. L., Adams, R. J., Wilson, M. R., \& Haldane, S. (2007). ConQuest, Version 2.0 [Computer software]. St. Paul, MN: Assessment Software Corp.

Ying, Y. (1999). Strengthening intergenerational/intercultural ties in migrant families: A new intervention for parents. Journal of Community Psychology, 27, 89-96.

Received September 22, 2009

Revision received June 8, 2010

Accepted June 14, 2010

\section{Instructions to Authors}

For Instructions to Authors, please consult the February 2010 issue of the volume or visit www.apa.org/ pubs/journals/ccp and click on the "Instructions to authors" tab in the Journal Info box. 